\title{
Influence of polymer binder on the performance of diF-TES-ADT based organic field effect transistor
}

\author{
Tommaso Salzillo, ${ }^{\mathrm{a}, \mathrm{c}, *}$ Francesco D’Amico, ${ }^{\mathrm{b}}$ Nieves Montes, ${ }^{\mathrm{a}}$ Raphael Pfattner ${ }^{\mathrm{a}}$ and Marta Mas-Torrent ${ }^{\mathrm{a}, *}$
}

a. Institut de Ciència de Materials de Barcelona, ICMAB-CSIC, Campus UAB, 08193 Bellaterra, Spain. tommaso.salzillo@unibo.it (T.S.); mmas@icmab.es (M.M.)
b. Elettra Sincrotrone Trieste S.C.p.A., S.S. 14 km 163,5 in Area Science Park, 34149 Basovizza TS, Italy.
c. Department of Materials and Interfaces, Weizmann Institute of Science, 76100 Rehovot, Israel.

\begin{abstract}
The presented work concerns the study of solution sheared organic thin film transistors based on a 2,8-difluoro-5,11bis(triethylsilylethynyl)anthradithiophene (diF-TES-ADT) polymer blend. Four different polymer binders were tested (i.e., polystyrene 10K and 100K g/ $\mathrm{mol}$ and poly(methyl methacrylate) $25 \mathrm{~K}$ and $120 \mathrm{~K} \mathrm{~g} / \mathrm{mol}$ ) in order to investigate the influence of the polymer binder nature and its molecular weight on the thin film morphology and device performance. Structural analysis by X-ray and Raman spectroscopy combined with Atomic Force Microscopy (AFM) and polarized optical microscopy were used for a complete characterization of the thin films. Additionally, resonance Raman spectroscopy elucidated the isomeric composition of the thin film devices and the different interactions of the organic semiconductor with the polymer binders.
\end{abstract}

\section{Introduction}

Organic semiconductors (OSCS) are a class of functional materials with a wide range of physical and chemical properties. ${ }^{1}$ Their excellent versatility in terms of synthesis and processing led to the development of organic devices such as organic light-emitting diodes (OLEDs), photovoltaic cells (OPVs) and organic field-effect transistors (OFETs). ${ }^{2}$ Organic electronics cannot compete with the stringent requirements dictated by the current Si-based electronics, such as the high charge carrier mobility and the long-term stability. However, organic electronics can be successfully used in low-power and mechanical compliant devices due to the compatibility of OSCbased devices with flexible substrates. For this reason, both academic and industrial research efforts have been devoted to pushing forward organic electronics, which has resulted in important advances regarding: i) the synthesis of novel soluble organic semiconducting materials with high thermal and shelf stability for low cost solution processability, and ii) the enhancement of the device properties, such as charge transport, photoconductivity, electroluminescence and superconductivity. ${ }^{3}$

The fabrication of thin film OFETs through solution techniques is highly appealing for low cost, flexible and large area applications. Among these, bar-assisted meniscus shearing (BAMS, Figure 1) is a coating method that allows for the rapid formation of crystalline films over large-area substrates with high throughput. ${ }^{4}$ The BAMS technique has been often employed with blends of a small molecule organic semiconductor and an amorphous insulating polymer to promote the crystallisation of the semiconductor due to the vertical-phase separation that takes place during the thin film deposition, in which the polymer rests on the dielectric substrate and the OSC crystalline layer lies on top. The deposition parameters such as temperature, organic semiconductor/polymer ratio, solvent, deposition rate and nature and molecular weight of the blending agent can be used to control the morphology and the structure of the film.., 6
Molecular packing, phase homogeneity and morphology in these systems not only affect the charge transport properties of the OSC thin film, but also influences the device-to-device reproducibility. ${ }^{7}$ Different polymorphic structures are characterized by different molecular orientations and this is reflected into different molecular orbital overlaps, hence different charge transport properties. Within this context, polymorphism can be considered as an additional degree of freedom of the molecular system and thus, must be controlled. Fluorinated 5,11-bis(triethylsilylehtynyl) anthraditiophene (diFTES-ADT) is a benchmark organic small semiconductor molecule (Figure 1), which has shown excellent properties when employed in organic field-effect transistors (OFETs), namely high field-effect mobility and low threshold voltage..$^{8,9}$ Blends of diF-TES-ADT with polystyrene (PS) deposited by BAMS have been reported to give rise to devices exhibiting high

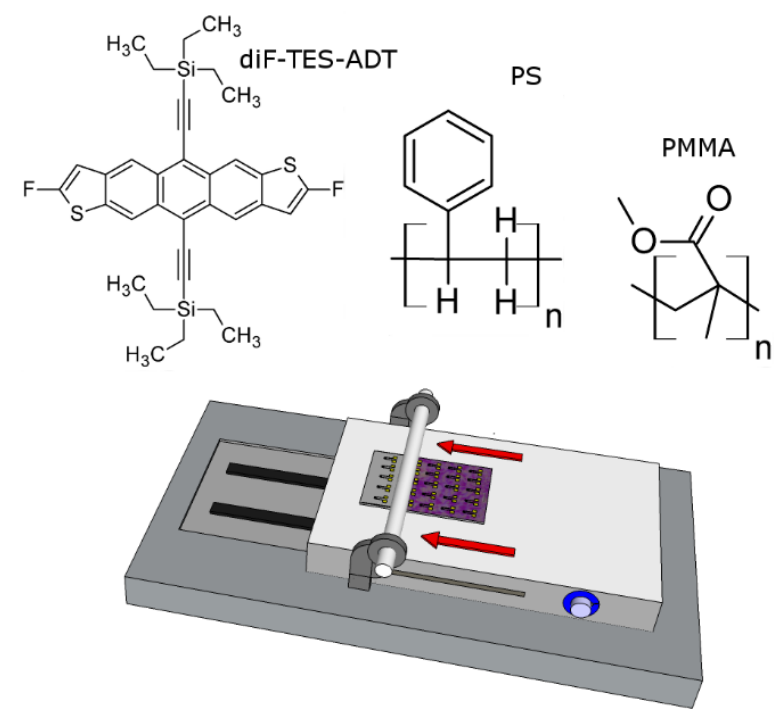

Figure 1 Top. Chemical structures of diF-TES-ADT, polystyrene (PS) and poly(methyl methacrylate) (PMMA). Bottom: Schematic representation of the BAMS apparatus. 
performance and stability with potential in sensing and bioapplications. ${ }^{4,10-12}$

Similarly to many other small molecules, various diF-TES-ADT polymorphs have been identified in the literature. ${ }^{13}$ Previously, two slightly different enantiotropic phases were resolved with their transition temperature at $21{ }^{\circ} \mathrm{C} .{ }^{13}$ Both enantiotropic polymorphs crystalize in P-1 space group showing layered structures with the molecules cofacially packed in a twodimensional $\pi$-stack. The differences in the tilt angle of ADT core plane are negligible and the main changes can be described in terms of interlayer and intralayer shifts, giving rise to a weak distortion of the unit cell with a detectable decrease in the interplanar distance in the HT polymorph. Recent studies performed in our lab have demonstrated that modifying the coating speed during the BAMS deposition of a blend of diF-TES$A D T: P S$, the formation of the low-temperature (LT) and hightemperature $(\mathrm{HT})$ phases can be modulated impacting on the device performance. ${ }^{14}$ This effect is due to the impact of the coating speed on the kinetics and thermodynamics of the crystallisation process. Typically, at low speeds the thermodynamic polymorph is favoured, while at higher speeds the formation of kinetic phases prevails. ${ }^{5}$ In order to gain a deeper understanding of the role of the polymer weight and polymer nature on the thin films morphology and structure, in this work we report the fabrication of OFETs based on blends of diF-TES-ADT with poly(methyl methacrylate) (PMMA) and polystyrene (PS) of different molecular weights. A complete characterization of the thin films morphology, spectroscopic and structural characteristics as well as their electrical properties were carried out. Our work demonstrates that the nature of the polymer does not have a strong influence on the polymorphic composition of the diF-TES-ADT films but, instead, plays a crucial role in the device performance, with the best performances achieved when the apolar PS binder polymer was used.

\section{Experimental}

All the films were deposited by BAMS technique, ${ }^{15}$ using solutions of diF-TES-ADT (Lumtec, purity >99\%) blended with various polymers in chlorobenzene ( $C B$, Sigma-Aldrich HPLC grade). As binding agents, Polystyrene (PS) of molecular weights $10 \mathrm{~K}$ and $100 \mathrm{~K} \mathrm{~g} / \mathrm{mol}$ (Sigma-Aldrich), and Poly(methyl methacrylate) (PMMA) of molecular weights $25 \mathrm{~K}$ (Polysciences Inc) and $120 \mathrm{~K} \mathrm{~g} / \mathrm{mol}$ (Sigma-Aldrich) have been employed. All the polymers were atactic. The solution concentration and OSC:polymer ratio were fixed at $2 \% \mathrm{w} / \mathrm{w}$ and $4: 1$, respectively, as reported in previous work. ${ }^{14}$ The temperature of the hot bed of the BAMS was kept constant at $105{ }^{\circ} \mathrm{C}$, and the shearing speed was varied from 0.1 to $10 \mathrm{~mm} / \mathrm{s}$. Before depositing the OSC layer, the gold electrodes were exposed for 25 minutes to ozone and then functionalized with pentafluorobenzothiol (PFBT, Sigma-Aldrich) by immersing the substrates for $15 \mathrm{~min}$ in a $2 \mu \mathrm{l} / \mathrm{ml}$ PFBT solution in isopropanol (IPA).

OFETs in bottom gate/bottom contact (BGBC) architecture were fabricated starting from $\mathrm{p}$-doped $\mathrm{Si} / \mathrm{SiO}_{2}$ wafers purchased from Si-Mat $\left(\mathrm{SiO}_{2}\right.$ of thickness $\left.200 \mathrm{~nm}, \mathrm{C}=17.25 \mathrm{nF} / \mathrm{cm}^{2}\right)$. Interdigitated electrodes with different channel lengths $(L=25$;
$50 ; 75 ; 100 \mu \mathrm{m}, \mathrm{W} / \mathrm{L}$ ratio kept at 100 ) were fabricated by means of photolithography (Micro-Writer ML3 from Durham Magneto Optics Ltd.) with a lateral resolution of $5 \mu \mathrm{m}$.

Polarized optical microscopy (POM) images were taken with Olympus BX51 microscope equipped with polarizer and analyzer at $90^{\circ}$ in reflection mode.

Film topography, thickness and roughness were extracted from atomic force microscopy (AFM) images (Keysight 5100 system from Agilent) taken in constant amplitude dynamic mode. The images were analyzed with the Gwyddion 2.52 software. The roughness was obtained as the root mean square (RMS). The film thickness was extracted by taking the profile perpendicular to a film scratch and fitting it to a step function.

Film crystallinity and polymorphic structure were characterized by X-ray diffraction (XRD) examining the out-of-plane diffraction peaks in $\Phi / 2 \Phi$ configuration (Siemens D-5000 diffractometer) employing $\mathrm{Cu}-\mathrm{K}_{\alpha 1}$ radiation $(\lambda=1.5406 \AA)$ at room temperature. The peaks were fitted to a Voigt function. ${ }^{16}$

The electrical performance was assessed under ambient conditions using an Agilent B1500A semiconductor parameter analyzer coupled with a Karl SüsS probe station. Transfer characteristics were measured both in the linear and saturation regimes, with $V_{D}$ fixed at $-5 \mathrm{~V}$ and $-15 \mathrm{~V}$, respectively, while $\mathrm{V}_{\mathrm{G}}$ was swept between $+5 \vee$ and $-20 \vee$ forward and backwards. Output characteristics were measured under constant $V_{G}=+5$, $-5,-15$ and $-25 V$, while $V_{D}$ was swept between $+5 V$ and $-25 V$, also forward and backwards. The mobility and threshold voltage were extracted for the linear and saturation regimes using the equations (1) and (2), both in the forward and backward sweeps, respectively.

$$
\begin{gathered}
\mu_{L I N}=\frac{L}{W C V_{D}}\left(\frac{\partial I_{D}}{\partial V_{G}}\right) \\
\mu_{S A T}=\frac{2 L}{W C}\left(\frac{\partial \sqrt{\left|I_{D}\right|}}{\partial V_{G}}\right)^{2}
\end{gathered}
$$

Resonant Raman scattering experiments using UV excitation were performed at BL10.2-IUVS beamline at Elettra-Sincrotrone Trieste. Raman spectra were recorded exciting with a diode laser tuned at $266 \mathrm{~nm}$, the power reaching the sample was 300 $\mu \mathrm{W}$, with a spot size of about $100 \mu \mathrm{m}$.. The signal was acquired in back-scattering geometry by means of a Czerny Turner spectrometer (TriVista 557, Princeton Instruments) equipped with a holographic reflection grating of 1800 lines $/ \mathrm{mm}$ optimized for UV radiation range. To avoid photo-degradation of the samples due to a prolonged UV irradiation, we performed measurements on an oscillating stage with a frequency of $1 \mathrm{~Hz}$, so that the real volume illuminated was continuously changing corresponding to a spot of $1 \mathrm{~mm}$ in length. The scattered signal was collected by a back-thinned Peltier cooled CCD detector (Princeton Instruments). The frequency spectra calibration was carried out by using a cyclohexane solution spectrum recorded in the same experimental condition. A detailed description of the experimental setup can be found in D'Amico et al. ${ }^{17}$ 


\section{Results and discussion}

OFETs based on thin films of diF-TES-ADT:binding polymer in a ratio 4:1 were deposited by BAMS at $105 \circ \mathrm{O}$ and $10 \mathrm{~mm} / \mathrm{s}$. As binding polymer, PS and PMMA of two comparable molecular weights were employed, keeping the other fabrication conditions (temperature and shearing speed) constant as described in the Experimental section. Characteristic parameters for devices prepared with low molecular weight and high molecular weight polymers were extracted both in linear and in saturation regime and compared. In all cases, statistics were performed on sets of at least 30 devices for each OSC:polymer formulation.

Figure 2 displays statistics on the mobility $(\mu)$ extracted in both linear and saturation regime and representative transfer characteristics for diF-TES-ADT blended with the two lower molecular weight polymers PS10K and PMMA25K. The average mobility $\left(\mu_{\mathrm{av}}\right)$ for the devices blended with PS10K results to be around $1 \mathrm{~cm}^{2} \mathrm{~V}^{-1} \mathrm{~s}^{-1}$ as previously reported. ${ }^{14}$ The $\mathrm{V}_{\mathrm{TH}}$ is slightly more negative in the reverse than in the forward sweep, which is probably related to charge trapping, and the $I_{\text {on/off }}$ ratio is estimated to be up to $10^{6}$. The off-current $\left(l_{\text {off }}\right)$ in reverse mode is almost 1-2 orders of magnitude higher compared to forward sweep. Also, this can be attributed to residual polarization, i.e. charge trapping.

However, when dif-TES-ADT is blended with PMMA25K, the field-effect mobility drops by 20-30 times with respect to the PS10K samples. Importantly, it should be highlighted that a higher contact resistance is detected for these blends, as indicated by the "S-shaped" curve in the low-voltage range of their output characteristics (Figure S1). Further, the transfer in the linear regime also reveals that these devices are less ideal and deviate more from the theoretical model than PS10K-based OFETs. Indeed, while in the PS devices, $I_{D}$ vs $V_{G}$ reveals quite a good linearity in the low-voltage range, in the PMMA ones $I_{D}$ deviates much more from linearity. This common non-ideal behaviour has been already encountered in OFETs and it can be caused by several effects, such as contact resistance and charge traps. ${ }^{18,19}$ Therefore, in the PMMA devices the linear fit has been adjusted, approximately, in the middle part of the curve. Noticeably, in the saturation regime the devices show a more ideal behaviour. Further, a larger clockwise hysteresis is observed in the PMMA blends, which can be attributed to a larger charge trapping, which is ascribed to the more polar nature of this polymer. $V_{\mathrm{TH}}$ extracted from transfer characteristics on average gives a positive value for the forward sweep and a negative one for the reverse one, while $I_{\text {on/off }}$ ratio decreases two orders of magnitude (i.e., $10^{4}$ ) with respect to the devices based on PS10K.

Analogous studies were performed using the higher PS and PMMA molecular weight polymers and the same experimental conditions. In Figure 3 the mobility statistics for the OFETs based on the higher molecular weight PS100K and PMMA120K are reported.

As for the devices based on the lower molecular weight polymer binders, the performance found when PS100K is used is enhanced compared to when PMMA120K is employed. However, in the case of PS, the device mobility is decreased with the highest molecular weight polymer to one third of the value achieved with the lowest polymer weight PS. In contrast, the polymer weight is not affecting so significantly the mobility of the PMMA-based devices, and even a slightly higher mobility is found in the films based on the highest molecular weight PMMA. Table 1 summarizes the average performance parameters obtained for each sample set, under linear and saturation conditions for both the forward and reverse sweeps.

Table 1 Average mobility and threshold voltage in the linear and saturation regimes for each sample set, sorted by polymer binder and sweep.

\begin{tabular}{|c|c|c|c|c|c|}
\hline \multirow{2}{*}{ Polymer binder } & & \multicolumn{2}{|c|}{ Forward } & \multicolumn{2}{c|}{ Reverse } \\
\cline { 2 - 6 } & & $\begin{array}{c}\mu \\
\left(\mathrm{cm}^{2} / \mathrm{V} \mathrm{s}\right)\end{array}$ & $\begin{array}{c}\mathrm{V}_{\mathrm{TH}} \\
(\mathrm{V})\end{array}$ & $\begin{array}{c}\mu \\
\left(\mathrm{cm}^{2} / \mathrm{V} \mathrm{s}\right)\end{array}$ & $\begin{array}{c}\mathrm{V}_{\mathrm{TH}} \\
(\mathrm{V})\end{array}$ \\
\hline \multirow{2}{*}{ PS 10K } & Lin. & $1.1 \pm 0.3$ & $-2.6 \pm 0.3$ & $1.2 \pm 0.3$ & $-3.6 \pm 0.4$ \\
\cline { 2 - 6 } & Sat. & $1.3 \pm 0.2$ & $-0.6 \pm 0.2$ & $1.4 \pm 0.3$ & $-1.5 \pm 0.4$ \\
\hline \multirow{2}{*}{ PMMA 25K } & Lin. & $0.04 \pm 0.01$ & $1.1 \pm 0.7$ & $0.04 \pm 0.01$ & $-1.1 \pm 0.7$ \\
\cline { 2 - 6 } & Sat. & $0.06 \pm 0.01$ & $1.8 \pm 0.4$ & $0.06 \pm 0.01$ & $0.2 \pm 0.6$ \\
\hline \multirow{2}{*}{ PS 100K } & Lin. & $0.4 \pm 0.2$ & $-2.6 \pm 0.6$ & $0.3 \pm 0.2$ & $-4.0 \pm 0.8$ \\
\cline { 2 - 6 } & Sat. & $0.4 \pm 0.2$ & $-0.6 \pm 0.6$ & $0.4 \pm 0.2$ & $-3.1 \pm 1.0$ \\
\hline \multirow{2}{*}{ PMMA 120K } & Lin. & $0.06 \pm 0.01$ & $-3.3 \pm 1.0$ & $0.06 \pm 0.01$ & $-4.3 \pm 1.0$ \\
\cline { 2 - 6 } & Sat. & $0.09 \pm 0.02$ & $-2.6 \pm 0.8$ & $0.09 \pm 0.09$ & $-3.3 \pm 0.9$ \\
\hline
\end{tabular}

Comparison of the POM and AFM topography images of the diFTES-ADT blends with the low (top) and high (bottom) molecular weight polymers are shown in Figure 4. Films fabricated using both PS10K and PS100K, show the same morphology appearing as large petal-like crystallites with clear boundaries that can be appreciated also in the AFM topography. The same type of morphology has been described in previous reports of films made by BAMS ${ }^{4,14}$ or by spin-coating with blends of diF-TES-ADT with PS. ${ }^{20}$ In these films, the grains appear smaller in the device channel area (interdigitated gold contacts) than on the bare $\mathrm{SiO}_{2}$ substrate, most probably due to higher nucleation density caused by the PFBT-treated electrodes. The proposed interactions that induce the nucleation of diF-TES-ADT on the electrodes are attributed to $\mathrm{F} \cdots \mathrm{F}$ and $\mathrm{F} \cdots \mathrm{S}$, as described by Ward et al. ${ }^{21}$ Nevertheless, between the two molecular weight PS, the $10 \mathrm{~K}$ seems to favour the formation of larger crystal domains, 

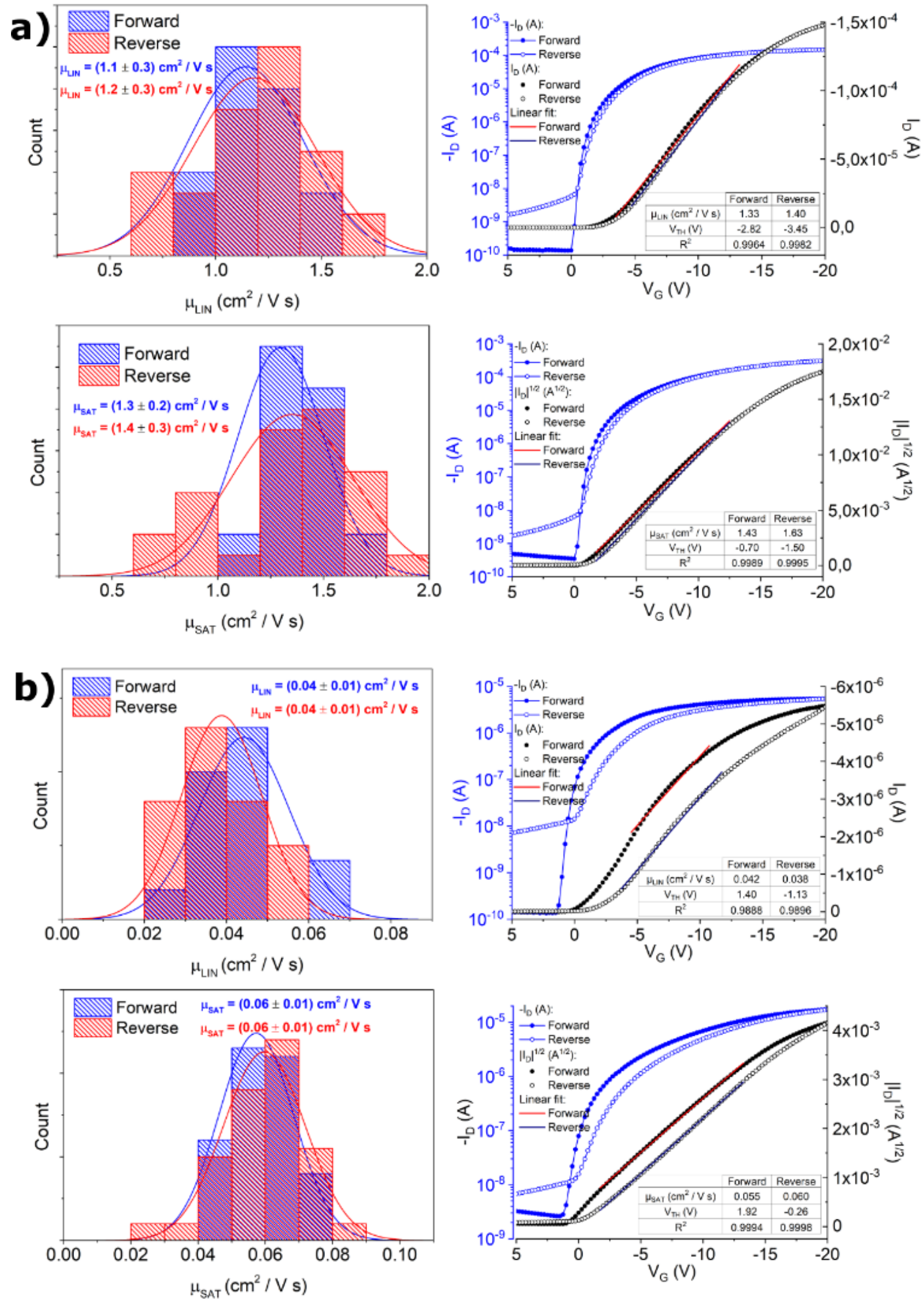

Figure 2 Mobility ( $\mu$ ) statistics for linear and saturation regimes for PS10K (a) and PMMA25K (b) based devices and corresponding representative transfer characteristics curves.

which also is in accordance with the higher charge carrier mobility found in these films. When we change the blending agent with PMMA we observe different features compared to the previous case. The films show a fine morphology instead of large petals (Figure 4 right side). The crystal domains appear much more irregular and with a dendritic structure with little differences in the channel region and on the bare $\mathrm{SiO}_{2}$ area, which might indicate that diF-TES-ADT is not strongly interacting with PFBT in these blends. Similarly to the PS-based 
films, the morphology does not change significantly with the molecular weight of the polymer binder.

From the AFM topography images and high profile analysis of scratched films, we observe that diF-TES-ADT:PMMA films are rougher compared with the PS blended ones. Indeed, the PS10K blended films show a thickness and root mean square (RMS) roughness of $19 \pm 3$ and $4 \mathrm{~nm}$, respectively, while PMMA25K samples tend to be thicker (of the order of $150 \mathrm{~nm}$ ), although
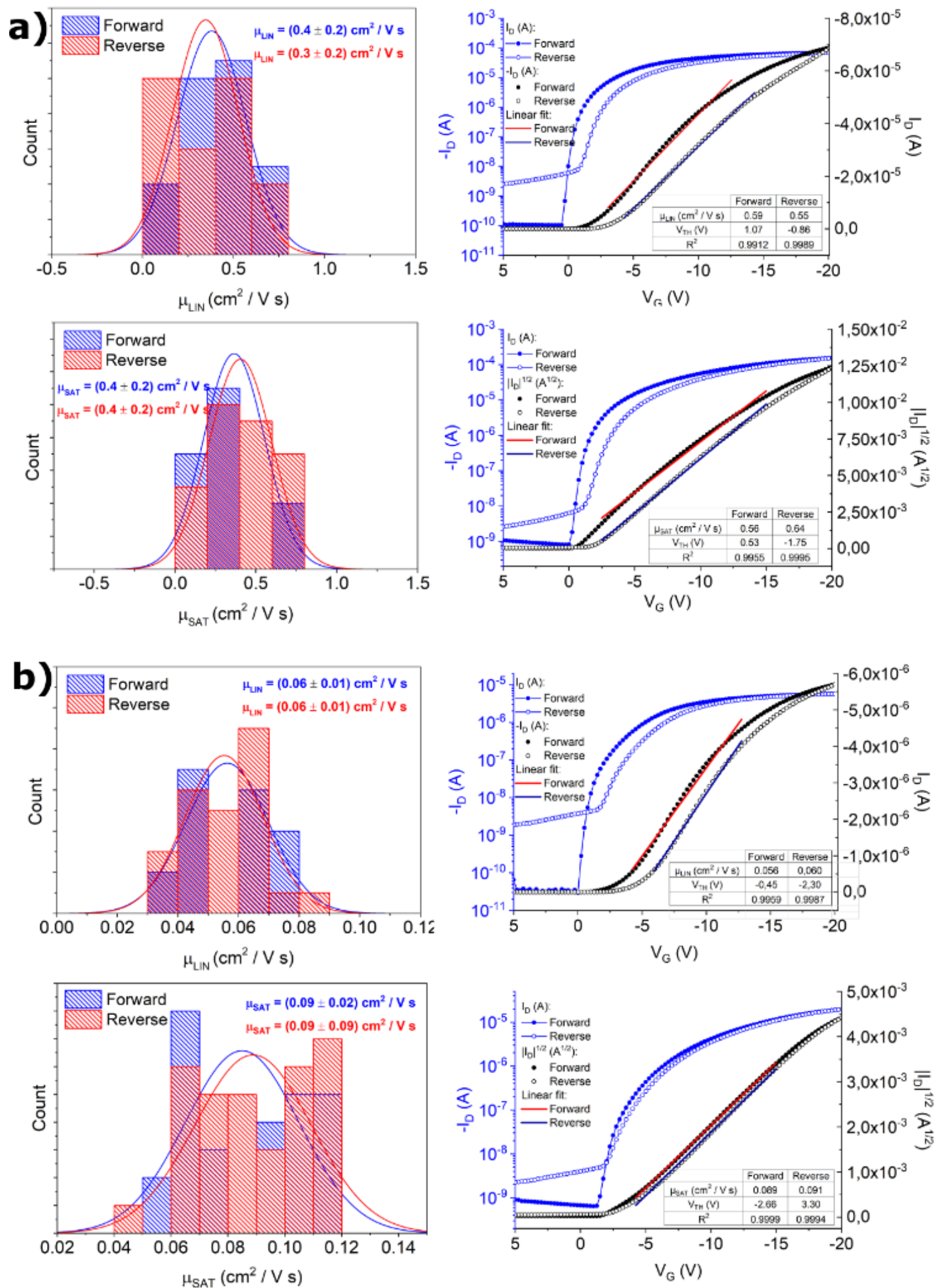

Figure 3 Mobility $(\mu)$ statistics for linear and saturation regimes for PS100K (a) and PMMA120K (b) based devices and corresponding representative transfer characteristics curves. 
due to its unusually elevated roughness of approx. $35 \mathrm{~nm}$, it is difficult to quantify the film thickness with the fitting procedure. Films with higher molecular weight polymers follow the same behaviour showing higher roughness for PMMA120K and smoother morphology for PS100K. Nevertheless, while for PMMA120K the average thickness resembles that of the PMMA25K, for PS100K a thicker film in the order of $40 \mathrm{~nm}$ is formed. This increased film thickness could be detrimental to the device performance.

The different morphology and roughness of the films can be the origin of the lower performance found in the PMMA-based OFETs. In previous spin-coated films of diF-TES-ADT with PMMA it was also observed that the resulting films exhibited high roughness. ${ }^{22}$ Differences related to a different vertical phase separation taking place cannot be ruled out, although this study is beyond the objective of the present work.

Under standard synthetic procedures diF-TES-ADT is obtained as a hardly separable mixture of syn and anti isomers, which differ between them from the relative position of the two sulphur atoms of the external aromatic rings. ${ }^{23}$ Due to the cost and time consuming procedure to separate the two isomers, in most of the works based on this OSC the isomeric mixture is used. ${ }^{10-12}$ Recently, it has been reported that employing the isomeric mixture decreases the OFET performances, which was attributed to the intrinsic molecular disorder. Additionally, between the two pure isomers the best characteristics were found with the pure anti. ${ }^{24} \mathrm{~A}$ complete Raman and theoretical, at density functional theory (DFT) level, study to probe the two isomers in drop-casted samples has been performed by Huang et al. ${ }^{25}$ Raman spectroscopy in $\pi-\pi$ conjugated systems has been proved to be a powerful tool for phase and chemical structure identification up to micrometric scale in confocal microscopy configuration. ${ }^{26,27}$
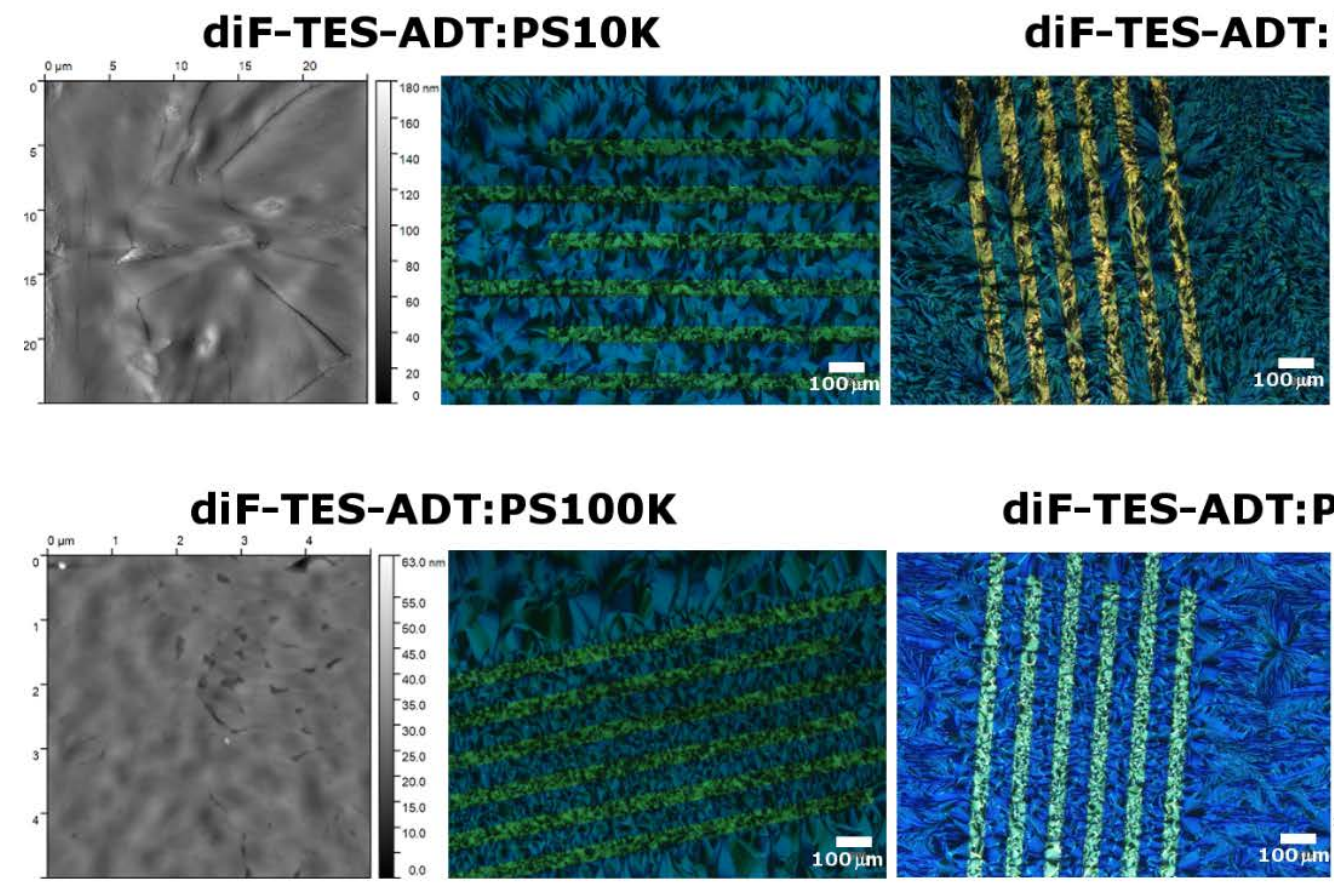

The thicknesses of the active semiconducting crystalline layers of the blended films fabricated by BAMS are very thin (previously reported to be in the order of $10-20 \mathrm{~nm}$ ), ${ }^{28,29}$ which makes a Raman characterization by a lab-based standard setup challenging. To characterize spectroscopically in situ the fabricated devices we performed UV Resonant Raman (UVRR) using the IUVS facility available at the ELETTRA synchrotron in Trieste (Italy), which allows for UV excitation. The use of RR measurements using excitation wavelengths into the deep ultraviolet (UV) spectral region enhances the signal of vibrational modes of conjugated organic molecules in resonance with the electronic transitions of the aromatic rings in the range of 200-300 $\mathrm{nm}$. In addition to the generally large resonance excitation enhancement, UV excitation has the further advantage that the light scattering efficiency scales with the fourth power of frequency and, moreover, the interference of the relaxed emission (photoluminescence) is reduced.

In Figure 5 (panel A) the representative spectra of the diF-TESADT blended films with PS10K and PMMA25K compared with the spectrum of a film prepared in the same conditions but based on only the OSC and the spectrum of the powder of the commercial as purchased OSC are reported. The spectral lineshape is characterized by several sharp peaks as typically occurs in crystals. More specifically, the peaks in the wavenumber range between 1100 and $1700 \mathrm{~cm}^{-1}$ coincide with the ones found by Huang and coworkers in diF-TES-ADT. ${ }^{25}$ Looking into detail, the peaks at $1143 \mathrm{~cm}^{-1}$ and $1187 \mathrm{~cm}^{-1}$ are assigned to C-

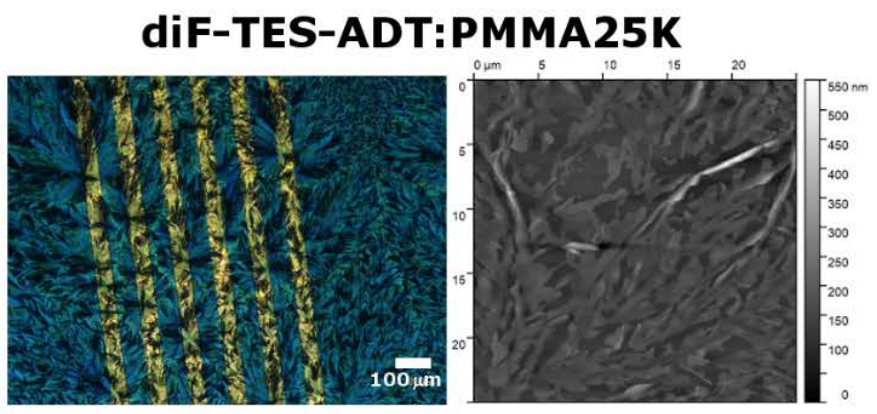

Figure 4 POM and AFM topography images for all the diF-TES-ADT blend films fabricated. 


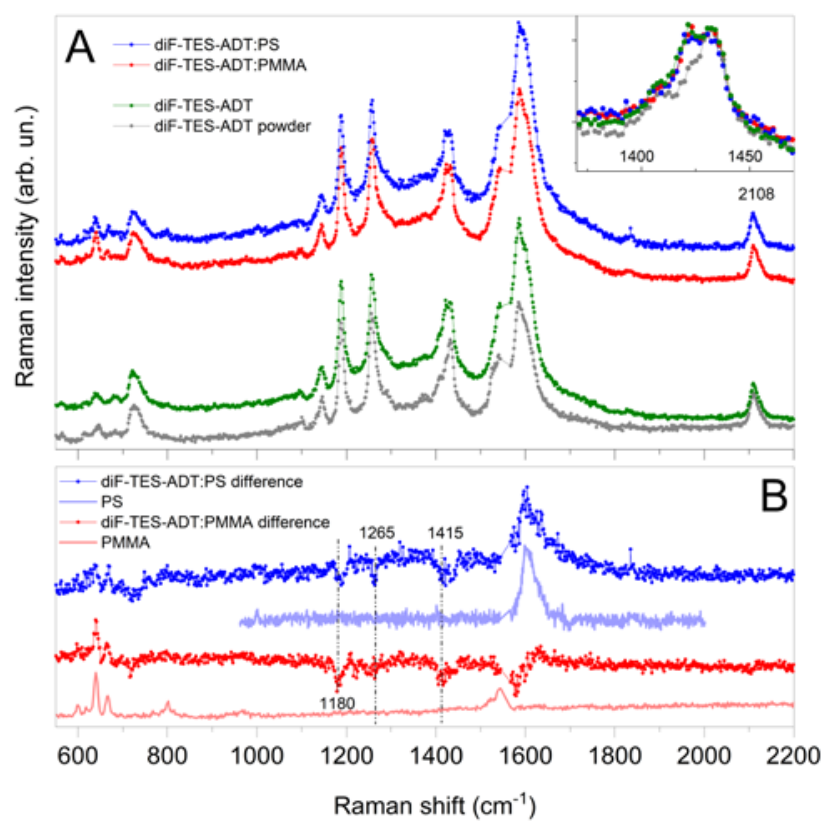

Figure 5 Panel A: Resonant Raman spectra, obtained with $266 \mathrm{~nm}$ laser excitation, of bare diF-TES-ADT thin film prepared by BAMS and diF-TES-ADT blended films with PS10K and with PMMA25K. The spectrum of the diF-TES-ADT commercial purchased powder is reported for comparison. The upper inset shows the same spectra in the wavenumber range of $1350-1500 \mathrm{~cm}^{-1}$. Panel B: Spectral difference between the film of the bare semiconductor and the blended one with PS10K (blue dotted curve) and with PMMA25K (red dotted curve). Spectra of PS and PMMA are reported for comparison.

$\mathrm{H}$ bending normal modes, while the peak at $1256 \mathrm{~cm}^{-1}$ is a ring breathing. The band between 1370 and $1470 \mathrm{~cm}^{-1}$ (enhanced in the top Panel of Figure 5) includes four overlapped peaks (1407, 1421,1434 and $1436 \mathrm{~cm}^{-1}$ ) associated to the C-C stretching of the short axis ring. Huang and coworkers ${ }^{25}$ evidenced how the peak at $1407 \mathrm{~cm}^{-1}$ is present only for the syn form, while the peak at $1421 \mathrm{~cm}^{-1}$ is typical of the anti isomer. Consequently, this spectral region can be used as a fingerprint to evaluate the syn or anti character of the blended films. The top panel of Figure 5 clearly shows that the peak at $1421 \mathrm{~cm}^{-1}$ is more pronounced in the diF-TES-ADT films (both blended and pristine) than in the commercial as purchased OSC powder, indicating an anti character of all the deposited films.

In order to investigate the interaction between diF-TES-ADT and both PMMA and PS binding polymers, in panel $B$ of Figure 5 we report the difference between the diF-TES-ADT:PMMA and the diF-TES-ADT film spectrum (red dotted curve) as well as the difference between diF-TES-ADT:PS and diF-TES-ADT films (blue dotted curve). Before carrying out the spectral subtraction all the spectra have been normalized in intensity to the peak at $2108 \mathrm{~cm}^{-1}$, which can be assigned to the $\mathrm{C} \equiv \mathrm{C}$ stretching on the chain outside the aromatic ring portion. ${ }^{30,31}$ To allow a correct spectral comparison panel $B$ of Figure 5 includes also the pristine spectra of PMMA (blue curve) and PS (red curve). Now we focus our attention to the spectral region in the 1100-1500 $\mathrm{cm}^{-1}$ wavenumber range. Although this region does not include any vibrational feature relative to PS and PMMA, it is possible to observe small spectral differences between the pristine and both the PS and PMMA blended forms. In particular the main differences are found near the $\mathrm{C}-\mathrm{H}$ bending peaks of the central
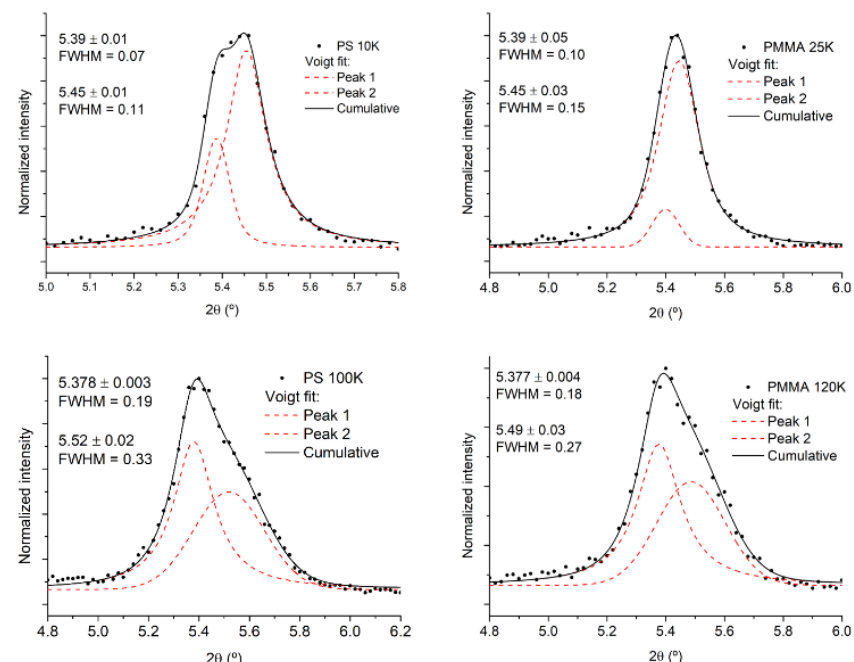

Figure 6 XRD (001) peak fits of the films fabricated with PS (10K and 100K) and PMMA (25K and 120K).

aromatic part $\left(1180 \mathrm{~cm}^{-1}\right)$, the ring breathing peak $\left(1256 \mathrm{~cm}^{-1}\right)$ and the $\mathrm{C}-\mathrm{C}$ stretching inside the aromatic rings. It is well known that the UV Raman cross section is modified in aromatic compounds when the polarizability of the micro environment changes. ${ }^{32-35}$ This occurs because such changes modify the molecular electronic states, which drive strongly the Raman cross section in resonance conditions. Although a quantitative estimation of this cross section change is difficult to be carried out, on the basis of these considerations, we can speculate that PS and PMMA interact with diF-TES-ADT through their aromatic parts in a slightly different way.

To complete the films characterization, X-Ray diffraction was performed analyzing in detail the shift of the (001) peaks to probe the polymorphic composition, as it has been previously reported. ${ }^{13,14}$ Indeed, the (001) peak of the LT polymorph is found at around $5.39^{\circ}$, while the peak of the HT phase appears at a higher value around $5.45^{\circ}$. In Figure 6 the deconvolution of the (001) diffraction peak of the prepared films are reported.

For all the samples a concomitant presence of the two polymorphs is detected in agreement with the results previously reported by Salzillo et al. ${ }^{14}$ for the solution shearing speed used in this work. This behaviour seems to be independent from the nature of the polymer binder. However, it can be noticed that with the higher molecular weight polymers the proportion of the two polymorphs is more similar, whereas the films with the lower molecular weight polymers are more enriched with the HT phase, especially in the films with the PMMA binding polymer. In our previous work, it was found that at lower coating speeds (i.e., $0.1 \mathrm{~mm} / \mathrm{s}$ ) the LT polymorph was dominant, and the HT phase was gradually formed by increasing the coating speed. However, the best performances were found at this intermediate speed of 10 $\mathrm{mm} / \mathrm{s}$ despite having a mixture of phases, which was attributed to the smoother and thinner films obtained in these conditions. Here, independently from the polymer binder nature and polymer weight, a similar behaviour was found (Figure S3).

In Figure S4 the full X-ray spectra of the films based on the different OSC:polymer formulations are reported not normalized. It can be observed that even if the signals are 
comparable, the widths of the diffraction peaks are clearly smaller for the OFETs based on the lower molecular weight PS10K and PMMA25K, in agreement with larger crystal domain.

\section{Conclusions}

In conclusion, we reported the use of blends of diF-TES-ADT and binding polymers as the active material in organic field-effect transistors. Thin films have been prepared employing the BAMS technique, which is compatible with high throughput and large scale applications. In particular, we explored the use of two binding polymers (PS and PMMA) of two different molecular weights and we found that they have a strong influence on the morphological and structural characteristics of the resulting thin films. Regarding the polymer binder nature, it was found that blends with PMMA give rise to rougher films with smaller crystalline domains compared with the blends of PS. Electrically, the best performances were found with the blends with PS, which display an enhanced mobility and a lower hysteresis attributed to the less polar character of this polymer that disfavours charge trapping. Taking into account the molecular weight, small differences were found with the PMMA films, however, a significant improvement in mobility was observed in the PS films of lower molecular weight, giving devices with mobility values above $1 \mathrm{~cm}^{2} \mathrm{~V}^{-1} \mathrm{~s}^{-1}$.

An in depth study of the structural properties of the samples has revealed that in all the samples the anti isomer seems to be dominant. Further, it has been found that the polymorphic composition of the films is mostly influenced by the shearing speed rather than by the polymer binder nature (Figure S3). All formulations sheared at $10 \mathrm{~mm} / \mathrm{s}$ yield a mixture of the two polymorphic structure of diF-TES-ADT as reported before. ${ }^{14}$ Thus, the variations in electrical performances cannot come from structural differences but, instead, from morphological factors, such as thin film smoothness and homogeneity and crystalline domain sizes, derived from the interactions of the OSC and the polymer between them and the interfaces during the crystallization process. Future work will be devoted to gaining a further understanding on the influence of the polymer and molecular weight on the vertical phase segregation occurring in the films.

\section{Conflicts of interest}

"There are no conflicts to declare".

\section{Acknowledgements}

This work was funded by DGI (Spain) project FANCY CTQ201680030-R and GENESIS PID2019-111682RB-I00, the Generalitat de Catalunya (2017-SGR-918), and the Spanish Ministry of Economy and Competitiveness, through the "Severo Ochoa" Programme for Centers of Excellence in R\&D (FUNFUTURE CEX2019-000917-S)). T.S. acknowledges the H2020-MSCACOFUND-2014 Programme (P-SPHERE, Grant agreement 665919). The authors acknowledge the Central European Research Infrastructure Consortium (CERIC-ERIC) consortium

for the access to experimental facilities at the ElettraSincrotrone Trieste, (Proposal number: 20187028).

\section{Notes and references}

2

C. Wang, H. Dong, L. Jiang and W. Hu, Chem. Soc. Rev., 2018, 47, 422-500.

E. J. Meijer, D. M. De Leeuw, S. Setayesh, E. Van Veenendaal, B. H. Huisman, P. W. M. Blom, J. C. Hummelen, U. Scherf and T. M. Klapwijk, Nat. Mater., 2003, 2, 678-682.

O. D. Jurchescu, J. Baas and T. T. M. Palstra, Appl. Phys. Lett., 2004, 84, 3061-3063.

I. Temiño, F. G. Del Pozo, M. R. Ajayakumar, S. Galindo, J. Puigdollers and M. Mas-Torrent, Adv. Mater. Technol., 2016, 1, 1600090.

S. Galindo, A. Tamayo, F. Leonardi and M. Mas-Torrent, Adv. Funct. Mater., 2017, 27, 1700526.

A. Tamayo, S. Riera-Galindo, A. O. F. Jones, R. Resel and M. Mas-Torrent, Adv. Mater. Interfaces, 2019, 6, 1900950. C. Qian, J. Sun, L. Zhang, H. Huang, J. Yang and Y. Gao, J. Phys. Chem. C, 2015, 119, 14965-14971.

Z. A. Lamport, K. J. Barth, H. Lee, E. Gann, S. Engmann, H. Chen, M. Guthold, I. McCulloch, J. E. Anthony, L. J. Richter, D. M. DeLongchamp and O. D. Jurchescu, Nat. Commun., 2018, 9, 5130.

J. Panidi, A. F. Paterson, D. Khim, Z. Fei, Y. Han, L. Tsetseris, G. Vourlias, P. A. Patsalas, M. Heeney and T. D. Anthopoulos, Adv. Sci., 2018, 5, 1700290.

A. Kyndiah, F. Leonardi, C. Tarantino, T. Cramer, R. MillanSolsona, E. Garreta, N. Montserrat, M. Mas-Torrent and G. Gomila, Biosens. Bioelectron., 2020, 150, 111844.

Q. Zhang, F. Leonardi, R. Pfattner and M. Mas-Torrent, $A d v$. Mater. Interfaces, 2019, 6, 1900719.

S. Ricci, S. Casalini, V. Parkula, M. Selvaraj, G. D. Saygin, P. Greco, F. Biscarini and M. Mas-Torrent, Biosens.

Bioelectron., 2020, 167, 112433.

O. D. Jurchescu, D. A. Mourey, S. Subramanian, S. R. Parkin, B. M. Vogel, J. E. Anthony, T. N. Jackson and D. J. Gundlach, Phys. Rev. B - Condens. Matter Mater. Phys., 2009, 80, 085201.

T. Salzillo, N. Montes, R. Pfattner and M. Mas-torrent, J. Mater. Chem. C, 2020, Advanced article 10.1039/DOTC03222K.

F. G. Del Pozo, S. Fabiano, R. Pfattner, S. Georgakopoulos, S. Galindo, X. Liu, S. Braun, M. Fahlman, J. Veciana, C. Rovira, X. Crispin, M. Berggren and M. Mas-Torrent, Adv. Funct. Mater., 2016, 26, 2379-2386.

T. H. de Keijser, J. I. Langford, E. J. Mittemeijer and A. B. P. Vogels, J. Appl. Crystallogr., 1982, 15, 308-314.

F. D'amico, M. Saito, F. Bencivenga, M. Marsi, A. Gessini, G. Camisasca, E. Principi, R. Cucini, S. Di Fonzo, A. Battistoni, E. Giangrisostomi and C. Masciovecchio, Nucl. Instruments Methods Phys. Res. Sect. A Accel. Spectrometers, Detect. Assoc. Equip., 2013, 703, 33-37.

H. H. Choi, K. Cho, C. D. Frisbie, H. Sirringhaus and V. Podzorov, Nat. Mater., 2018, 17, 2-7. 

2014, 2, 245-255.

21 J. W. Ward, M. A. Loth, R. J. Kline, M. Coll, C. Ocal, J. E. Anthony and O. D. Jurchescu, J. Mater. Chem., 2012, 22, 19047-19053.

N. Shin, J. Kang, L. J. Richter, V. M. Prabhu, R. J. Kline, D. A. Fischer, D. M. Delongchamp, M. F. Toney, S. K. Satija, D. J. Gundlach, B. Purushothaman, J. E. Anthony and D. Y. Yoon, Adv. Funct. Mater., 2013, 23, 366-376.

23 R. K. Hallani, K. J. Thorley, Y. Mei, S. R. Parkin, O. D. Jurchescu and J. E. Anthony, Adv. Funct. Mater., 2016, 26, 2341-2348.

24 P. J. Diemer, J. Hayes, E. Welchman, R. Hallani, S. J. Pookpanratana, C. A. Hacker, C. A. Richter, J. E. Anthony, T. Thonhauser and O. D. Jurchescu, Adv. Electron. Mater., 2017, 3, 1600294.

C. W. Huang, X. You, P. J. Diemer, A. J. Petty, J. E. Anthony, O. D. Jurchescu and J. M. Atkin, Commun. Chem., 2019, 2, 22.

T. Salzillo, A. Rivalta, N. Castagnetti, S. D'Agostino, M. Masino, F. Grepioni, E. Venuti, A. Brillante and A. Girlando, CrystEngComm, 2019, 21, 3702-3708.

T. Salzillo, S. D'Agostino, A. Rivalta, A. Giunchi, A. Brillante, R. G. Della Valle, N. Bedoya-Martínez, E. Zojer, F. Grepioni and E. Venuti, J. Phys. Chem. C, 2018, 122, 18422-18431. F. Leonardi, S. Casalini, Q. Zhang, S. Galindo, D. Gutiérrez and M. Mas-Torrent, Adv. Mater., 2016, 28, 10311-10316. A. Pérez-Rodríguez, I. Temiño, C. Ocal, M. Mas-Torrent and E. Barrena, ACS Appl. Mater. Interfaces, 2018, 10, 72967303. S. Li, T. Chen, Y. Wang, L. Liu, F. Lv, Z. Li, Y. Huang, K. S. Schanze and S. Wang, Angew. Chemie - Int. Ed., 2017, 56, 13455-13458.

31 M. C. di Gregorio, L. J. W. Shimon, V. Brumfeld, L. Houben, M. Lahav and M. E. van der Boom, Nat. Commun., 2020, 11, 380. S. A. Oladepo, K. Xiong, Z. Hong, S. A. Asher, J. Handen and I. K. Lednev, Chem. Rev., 2012, 112, 2604-2628.

33 T. Miura, H. Takeuchi and I. Harada, Biochemistry, 1988, 27, 88-94. H. Takeuchi, Biopolym. - Biospectroscopy Sect., 2003, 72, 305-317.

35 H. Takeuchi, Y. Ohtsuka and I. Harada, J. Am. Chem. Soc., 1992, 114, 5321-5328. 